\title{
The role of chemical shift magnetic resonance imaging in differentiating osteoporotic benign and malignant vertebral marrow lesions
}

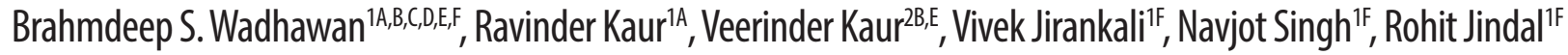 \\ 'Government Medical College and Hospital, Chandigarh, India \\ ${ }^{2}$ Government Medical College, Amritsar, India
}

\begin{abstract}
Purpose: To evaluate the usefulness of chemical shift imaging (CSI) in differentiating benign osteoporotic and malignant vertebral marrow lesions.

Material and methods: Patients undergoing spinal magnetic resonance imaging (MRI) for back pain, which showed altered marrow signal intensity on conventional MRI sequences, were included in the study. Patients with acute traumatic vertebral fractures, infective spondylodiscitis, paravertebral collections, etc. were excluded. The patients underwent CSI. In-phase and opposed-phase images were taken to calculate the signal intensity ratio (SIR) of the abnormal vertebra. The SIR of the mean signal intensity measured on opposed-phase to mean signal intensity measured on in-phase images was measured and recorded.
\end{abstract}

Results: The studied population included 30 patients, in whom 58 vertebrae were accessed, which included 38 dorsal, 18 lumbar, 1 sacral, and 1 cervical. Out of 58 vertebrae, 46 (79\%) were malignant and 12 (20\%) were benign. The mean CSI/SIR of malignant lesions was 0.96 and the mean SIR of benign lesions was 0.76 .

Conclusions: Conventional MRI sequences cannot always differentiate between benign and malignant lesions. So newer sequences like CSI have been developed. CSI SIR can be used as a new tool in differentiating benign osteoporotic and malignant vertebral marrow lesions.

Key words: chemical shift imaging, vertebral marrow lesion, in-phase/opposed-phase images.

\section{Introduction}

Vertebral marrow lesions are commonly seen in older age group of patients, due to a variety of causes ranging from infections, traumatic collapse, osteoporotic collapse, and neoplastic vertebral marrow changes $[1,2]$. Despite osteoporosis being one of the most common causes of vertebral collapse, metastasis in the spine is also very frequently seen, comprising about $39 \%$ of all bone metastases [1].

Many diseases show characteristic patterns of involvement of vertebrae, e.g. tubercular lesions cause paradiscal involvement with end plate erosions, pyogenic infections predominantly involve facet joints, neoplastic marrow infiltration involve vertebral body marrow with or without pedicles and sparing of end plates. However these findings may overlap; thus, there is a need for newer techniques that can differentiate various vertebral marrow lesions [3].

Standard radiographs (X-ray) are usually the initial imaging modality which is used to inspect the spine whenever a pathology is suspected. However, on standard radiographs it is difficult to determine the cause of a compression fracture and to see vertebral marrow lesions. Computed tomography (CT), on the other hand, is more dependable in revealing the calcifications and cortical outlines of bone as compared to X-ray. It can also delineate the extent of the tumour destruction more ef-

Correspondence address:

Dr. Brahmdeep S. Wadhawan, Government Medical College and Hospital, Chandigarh, India, e-mail: brahmdeep1@gmail.com

Authors' contribution:

A Study design · B Data collection · C Statistical analysis · D Data interpretation · E Manuscript preparation · F Literature search · G Funds collection 
fectively. However, it is not sensitive in the detection of, as well differentiating between, benign osteoporotic and malignant vertebral marrow lesions $[4,5]$.

Magnetic resonance imaging (MRI) is a good noninvasive modality for evaluation of bone marrow as well as for detection of marrow lesions. It can reveal subtle changes in physiological as well as pathological marrow composition in the form of signal alteration and thus can be helpful in knowing the cause of vertebral lesions [6].

Conventional MRI findings, which help in suggesting metastatic compression fracture, include abnormal signal intensity of the posterior elements, convex posterior vertebral body border, the presence of additional epidural mass or a paraspinal mass, and other spinal metastasis. The conventional MR techniques cannot always discriminate between benign osteoporotic and malignant vertebral marrow lesions (VML) because of their indistinguishable appearances. The limited specificity of these techniques results in invasive diagnostic modalities for accurate diagnosis [2,4-7]. Newer techniques like chemical shift imaging (CSI) have shown promising results for distinguishing between malignant and benign bone marrow lesions $[8,9]$.

CSI is based on the principle that the protons of water and fat are in the same phase at certain echo times (TE) and are in opposite phase at certain echo times. In the case of normal bone marrow, there will be a signal loss when out of phase because it contains protons of both fat and water [10]. But whenever there is some pathology like marrow infiltration by some neoplastic process in bone marrow, which effects the fat, it will be highlighted on apposed-phase imaging. The majority of neoplastic processes causes replacement of marrow fat, which in turn leads to the lack of normal signal dropout on out-of-phase images [2].

The purpose of this study is to evaluate the usefulness of chemical shift magnetic resonance imaging in differentiating benign osteoporotic and malignant vertebral marrow lesions.

\section{Material and methods}

This was a prospective analytical study conducted in a tertiary care hospital.

Patients were referred to the Department of Radiodiagnosis for MRI of the spine with clinical history of localized pain in the vertebrae with or without neurological symptoms or pain in the vertebral column in a patient of known malignancy.

Inclusion criteria were as follows: altered marrow signal intensity in one or more vertebrae on T1/T2/STIR; MRI sequences; collapsed vertebra with altered marrow signal intensity on T1/T2/STIR MRI sequences.

Exclusion criteria were as follows: acute traumatic vertebral fractures, known infective spondylodiscitis, paravertebral collections with endplate erosions on conventional MRI suggestive of infective spondylodiscitis, known ankylosing spondylitis and vertebral lesions treated with radiotherapy.
All MRI studies were performed on a 1.5 T MRI scanner (Achieva; Philips Medical Systems) with a spine array surface coil. The following MRI sequences were performed: routine conventional sequences, including T1-weighted imaging, T2-weighted turbo spin-echo imaging $(\mathrm{TR} / \mathrm{TE}=3100 / 120)$ in sagittal and axial planes, and short inversion time inversion-recovery (STIR) imaging $(\mathrm{TR} / \mathrm{TE}=4900 / 80)$ in the sagittal plane and coronal plane.

CSI in-phase and opposed-phase images were used to calculate the signal intensity ratio (SIR) of the abnormal vertebra. An ROI was applied over the abnormal area on in-phase images and copied into the computer memory to be pasted onto the opposed-phase images. The average value of signal intensity for 3 regions of interest was calculated and saved for both opposed-phase and in-phase sequences. The SIR of mean signal intensity measured on opposed-phase and in-phase images were measured.

The final clinical diagnosis was taken as the "gold standard" to characterize the vertebral lesions as benign or malignant. It was either based on biopsy results or on the results of radiological and clinical follow-up for a duration of at least 6 months.

\section{Statistical analysis}

Continuous data that were not normally distributed were reported as median and interquartile ranges. The distribution of the variables was tested with the Shapiro-Wilk test/ Kolmogorov-Smirnov tests of normality. Group comparisons of values of skewed data were made with the Mann-Whitney test for 2 groups (benign/malignant). For normally distributed data Student's $t$-test was applied to compare 2 groups. Categorical variables were reported as counts and percentages. Receiver operating characteristic (ROC) curves were calculated to find maximal cut-off value CSI/SIR levels. The ROC curve is a plot of sensitivity versus 1-specificity for maximal cut-off values. Sensitivity, specificity, positive predictive value, negative predictive value, and diagnostic accuracy values were calculated.

A $p$-value $<0.05$ was considered significant. Analysis was conducted using IBM SPSS STATISTICS (version 22.0).

\section{Results}

Thirty-two patients were included in this study. Two patients could not be followed up and were excluded from the study. Out of the remaining 30 patients, benign vertebral marrow lesions were seen in 8 patients and malignant vertebral marrow lesion in 22 patients. The total number of vertebrae involved in these patients was 58 , which included 38 dorsal, 18 lumbar, 1 sacral, and 1 cervical.

Out of these 58 vertebrae, 12 cases were of benign osteoporotic vertebral lesion, 30 of metastatic vertebral marrow lesions, 13 multiple myeloma, and 1 case each of plasmacytoma, Hodgkin's lymphoma, and primitive neuroectodermal tumour (PNET) (Table 1 and Figure 1). 
Table 1. Final diagnosis of benign and malignant vertebral marrow lesions

\begin{tabular}{|l|c|}
\hline Diagnosis & $n$ \\
\hline Benign osteoporotic & 12 \\
\hline Metastasis & 30 \\
\hline Multiple myeloma & 13 \\
\hline Primitive neuroectodermal tumour (PNET) & 1 \\
\hline Plasmacytoma & 1 \\
\hline Hodgkin's lymphoma & 1 \\
\hline
\end{tabular}

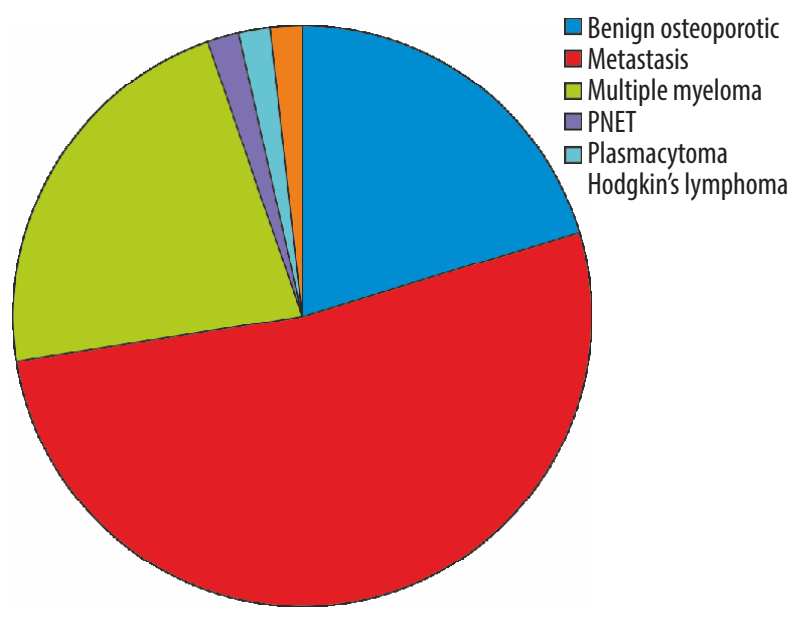

Figure 1. Final diagnosis of benign and malignant vertebral marrow lesions

The mean age of the patients was $59 \pm 12$ years. The mean age for malignant lesion was $57 \pm 11.5$ years, and that for benign lesions was $63 \pm 12.5$ years. There was no statistically significant difference between the age groups in differentiating benign and malignant vertebral marrow lesions $(p=0.290)$.

The studied population included 17 males and 13 females. There was no statistically significant difference between males and females in terms of involvement of benign and malignant vertebral lesions $(p=0.102)$.

On CSI, signal intensity ratio (SIR) of normal vertebrae was calculated in 27 vertebrae. The mean SIR of normal vertebrae was $0.306 \pm 0.144$.

The mean SIR on CSI of malignant vertebral lesions was $0.967 \pm 0.24$ and of benign vertebral lesions it was $0.76 \pm 0.235$ (Table 2).

When the CSI value was used in differentiating osteoporotic benign and malignant vertebral lesion, the area under the curve came was 0.758 and the $p$-value was 0.006 which was statistically significant. If the cut-off value of

Table 2. Mean signal intensity ratio (SIR) on chemical shift imaging of benign and malignant vertebral marrow lesions

\begin{tabular}{|l|c|c|}
\hline Final diagnosis & Number of vertebrae & Mean SIR \\
\hline Benign & 12 & 0.76 \\
\hline Malignant & 46 & 0.96 \\
\hline
\end{tabular}

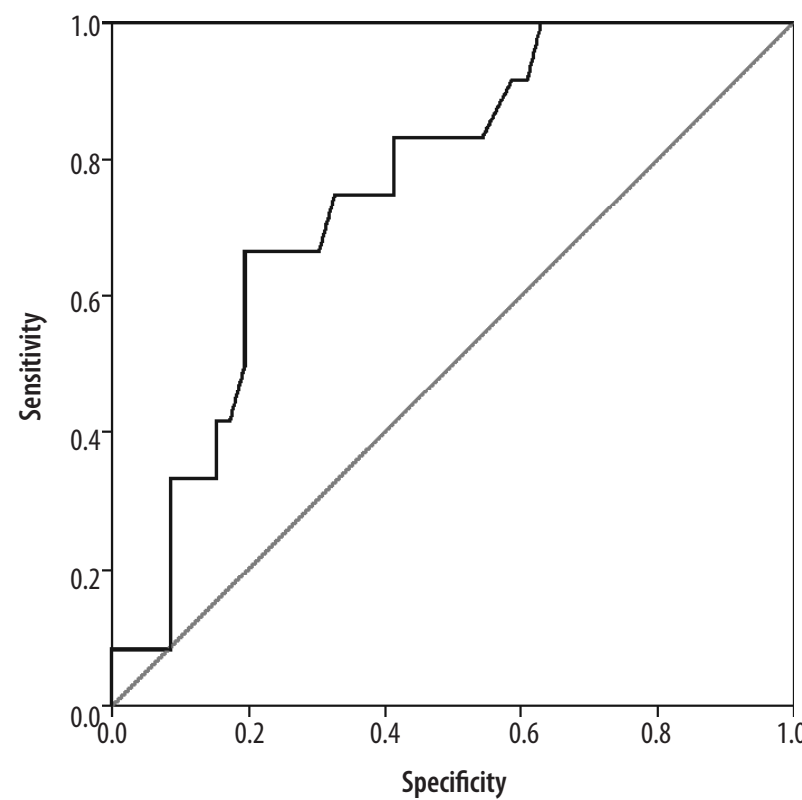

Figure 2. ROC curve

SIR on CSI was taken as 0.96 , then the sensitivity and specificity for differentiating benign osteoporotic and malignant lesions was $83.3 \%$ and $58.70 \%$, respectively, and the PPV was $34.48 \%$. The NPV was $93.10 \%$, and on biopsy it was proven to be CA lung-metastasis (Table 3).

\section{Discussion}

Vertebral marrow lesions are one of the most common pathologies seen in the elderly population. The causes include infections, traumatic collapse, osteoporotic collapse, and neoplastic vertebral marrow changes, out of which osteoporosis and metastasis are the major causes. The metastasis in spine contributes to nearly $39 \%$ of all bone metastases [1,2].

The initial imaging modality usually includes conventional radiography; however, many vertebral marrow lesions remain obscured on conventional radiography. CT can also be used for differentiation of these lesion. It is more dependable in revealing the calcifications and cortical outlines of bone as compared to X-ray. It can also delineate the extent of the tumour destruction more effectively. However, it is not very sensitive in the detection

Table 3. Cut-off signal intensity ratio (SIR), sensitivity, specificity, TPV, NPV, $p$-value, and area under curve

\begin{tabular}{|l|c|}
\hline Cut off value of SIR & 0.96 \\
\hline Senstivity & $83.3 \%$ \\
\hline Specificty & $58.70 \%$ \\
\hline TPV & 63.79 \\
\hline NPV & 93.10 \\
\hline$p$-value & 0.006 \\
\hline Area under curve & 0.758 \\
\hline
\end{tabular}


of, or in differentiating between, osteoporotic benign and malignant vertebral marrow lesions $[4,5]$.

MR imaging may be the initial modality for detection. Conventional MR imaging with sequences like T1 WI and T2 WI have proven to be useful in the differentiation of benign from malignant causes of vertebral lesions, but confident diagnosis is not always possible. Morphological features on $\mathrm{MR}$, such as abnormal signal intensity of the posterior elements, convex posterior vertebral body border, presence of additional epidural mass, or a paraspinal mass, suggest malignancy. Conventional MR techniques cannot always discriminate between benign and malignant vertebral marrow lesions (VML) because of their indistinguishable appearances. The limited specificity of these techniques results in invasive diagnostic modalities for accurate diagnosis [2,4-7]. Apart from conventional sequences, contrast enhanced MRI is also less effective in differentiating benign from malignant vertebral lesions because many benign lesions may also be enhanced after intravenous injection of contrast agent [11].

Differentiating a diffuse infiltrative lesion of marrow from the highly variable appearing normal hyper cellular haematopoietic red marrow has often faced diagnostic dilemmas. Hence, various sequences are being studied to more accurately differentiate between benign and vertebral marrow lesions. The present study was undertaken to evaluate the role of newer MR sequence CSI in differentiating benign osteoporotic from malignant vertebral marrow lesions.

In our study on 30 patients with ages ranging from 33 to 77 years and with a mean age of $59 \pm 12$ years, the mean age of those with malignant lesions was $57 \pm 11.5$ years and that of benign lesions was $63 \pm 12.5$ years. Out of 30 patients in our study 17 were males (56.6\%) and 13 were females (43.4\%).

The total number of vertebrae accessed in our study of 30 patients was 58, which included 38 dorsal, 18 lumbar, 1 sacral, and 1 cervical, i.e. in our study the most commonly involved vertebrae were dorsal followed by lumber.
CSI is one of the MRI techniques that can aid in differentiating benign and malignant vertebral lesions (Figure 3). In benign lesions, there is presence of normal fat in the marrow, which causes signal intensity suppression on opposed-phase images. On the other hand, in malignant lesions, the normal fat-containing marrow is replaced with tumour cells, which leads to a lack of signal suppression on the out-of-phase images $[12,13]$.

The mean signal intensity ratio (SIR) value of malignant lesions on CSI was 0.967 (Figures 4 and 5), and of benign lesions it was 0.76 (Figures 6 and 7). Using ROC analysis, SIR cut-off value of 0.96 was defined in our study to differentiate between benign osteoporotic and malignant vertebral marrow lesions. The area under the curve came was 0.758 and the $p$-value was 0.006 .

The CSI value in our study is in agreement with the CSI value reported by Tadros et al. [9], i.e. 0.94, but less than the cut off value reported by Ogura et al. [14], i.e. 1, and Zampa et al. [15], i.e. 1.2.

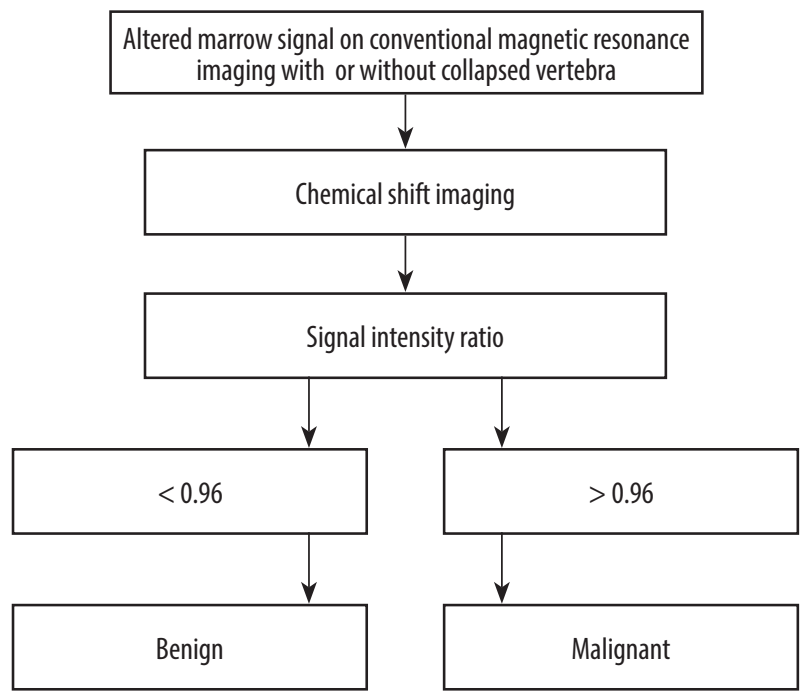

Figure 3. Schematic diagram representing role of chemical shift imaging in differentiating benign osteoporotic and malignant vertebral marrow lesions
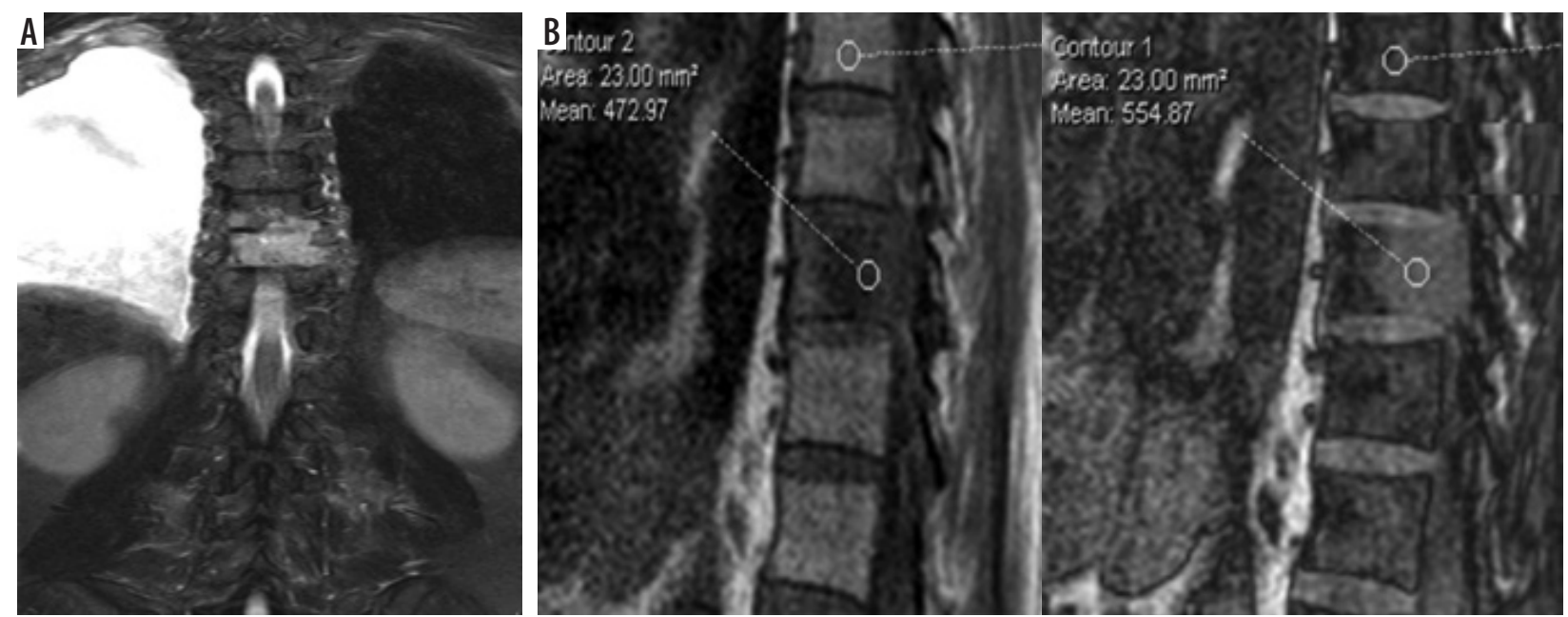

Figure 4. A) Coronal STIR images showing STIR hyperintensity at D9 vertebra. B) Sagittal chemical shift imaging (CSI) showing (SI in-phase and out-of-phase images of the same lesion in which signal intensity ratio was 1.17 

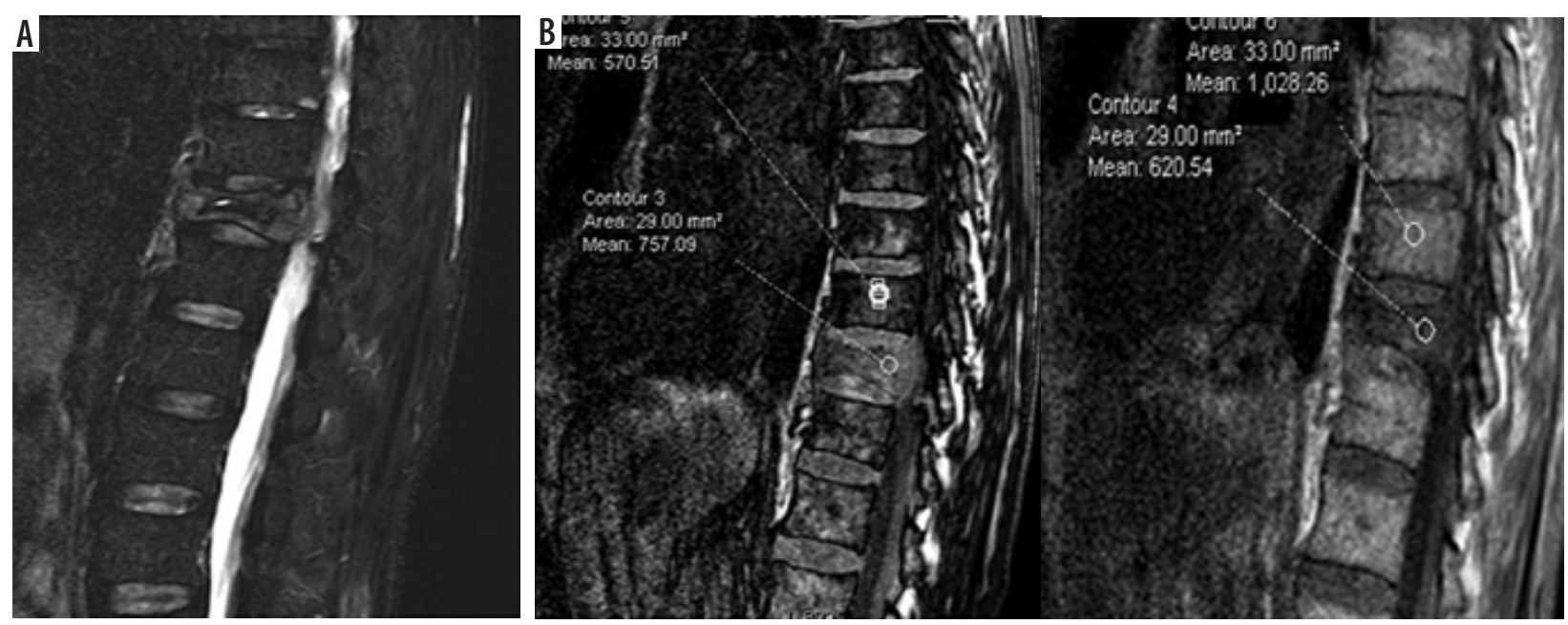

Figure 5. A) Sagittal STIR image-showing STIR hyperintensity and collapsed vertebra at D11 level. B) Sagittal chemical shift imaging (CSI) showing CSI in-phase and out-of-phase images of the same lesion in which signal intensity ratio was 1.02


Figure 6. A) Coronal STIR images-showing STIR hyperintensity and collapsed vertebra at L1. B) Sagittal chemical shift imaging (CSI) showing CSI in-phase and out-of-phase images of the same lesion in which signal intensity ratio was 0.22
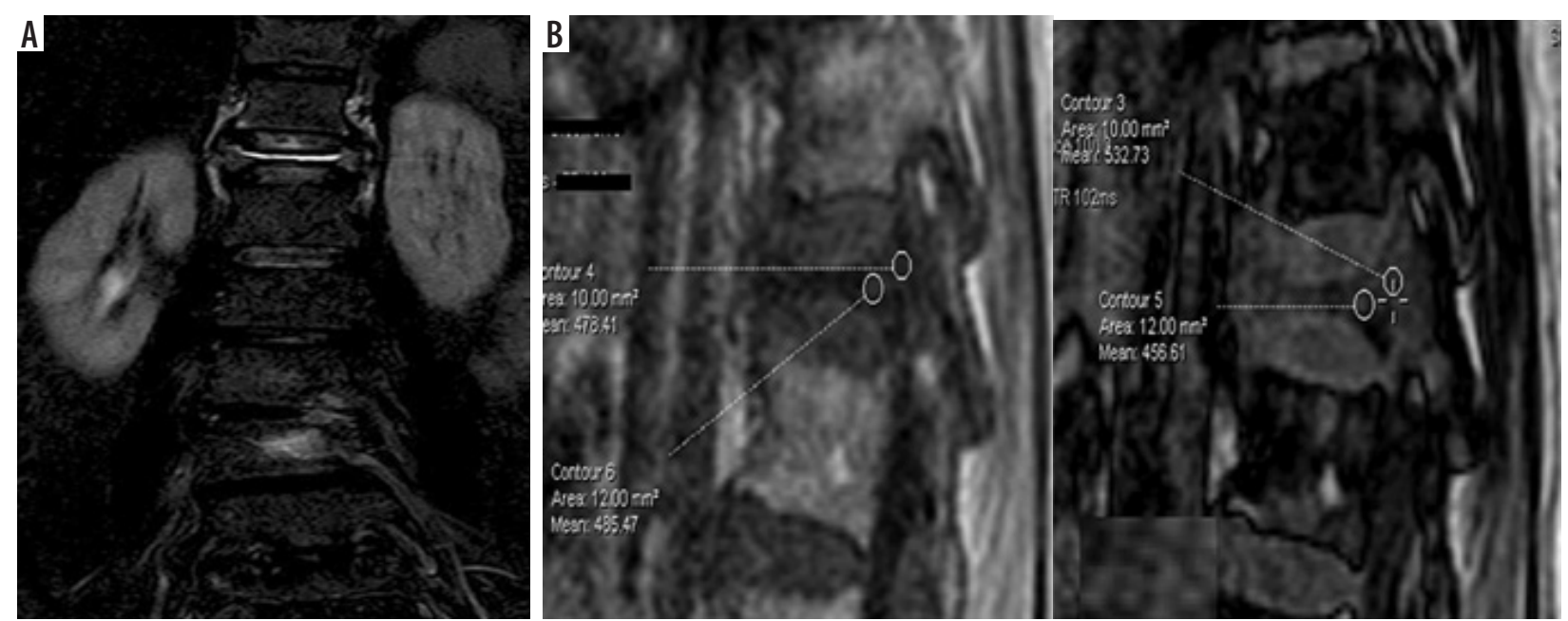

Figure 7. A) Coronal STIR-showing STIR hyperintensity and collapsed vertebra at D12. B) Sagittal chemical shift imaging (CSI) showing (SI in-phase and out-of-phase images of the same lesion in which signal intensity ratio was 0.6

In our study the cut-off of 0.96 for the SIR resulted in a sensitivity of $83.3 \%$ and specificity of $58.70 \%$, PPV of 34.8 , NPV of $93.10 \%$, and total predictive value of $63.7 \%$.
Our results are in concordance with the study done by Tadros et al. [9], who reported a sensitivity of $93 \%$ and specificity of $72 \%$. 
In a study done by Zidan et al. [16] in 32 patients, the SIR value on CSI was 0.91 , which had a sensitivity of $93 \%$ and specificity of $82 \%$ to differentiate benign from malignant vertebral lesions, which was higher than in our study.

Another study conducted by Erly et al. [17] in 25 patients, to study the role of CSI in differentiating benign and malignant lesions, reported a sensitivity of $95 \%$ and specificity of $89 \%$, which was higher than in our study.

Our study is also supported by Zampa et al. [15], who conducted their study on 86 patients to assess the role of CSI in differentiating benign and malignant vertebral marrow lesions. Their results showed a sensitivity of $88 \%$ and specificity of 80.49 .

\section{Conflict of interest}

The authors report no conflict of interest.

\section{Conclusions}

Our results showed that osteoporotic and malignant VML can be differentiated on CSI, which showed a statistically significant difference in these 2 groups. However, further studies are needed to prove the worth of CSI in differentiating benign osteoporotic and malignant lesions.

\section{References}

1. Jung HS, Jee WH, McCauley TR, et al. Discrimination of metastatic from acute osteoporotic compression spinal fractures with MR imaging. Radiogr Rev Publ Radiol Soc N Am Inc 2003; 23: 179-187.

2. Zajick DC, Morrison WB, Schweitzer ME, et al. Benign and malignant processes: normal values and differentiation with chemical shift MR imaging in vertebral marrow. Radiology 2005; 237: 590-596.

3. Turna O, Aybar MD, Tuzcu G, et al. Evaluation of vertebral bone marrow with diffusion weighted MRI and ADC measurements. Istanb Med J [Internet] 2014. Available from: http://www.istanbulmedicaljournal.org/eng/makale/699/22/Full-Text (Accessed: 11.10.2018)

4. Algra PR, Bloem JL, Tissing H, et al. Detection of vertebral metastases: comparison between MR imaging and bone scintigraphy. Radiogr Rev Publ Radiol Soc N Am Inc 1991; 11: 219-232.

5. Avrahami E, Tadmor R, Dally O, Hadar H. Early MR demonstration of spinal metastases in patients with normal radiographs and CT and radionuclide bone scans. J Comput Assist Tomogr 1989; 13: 598-602.

6. Hwang S, Panicek DM. Magnetic resonance imaging of bone marrow in oncology, Part 2. Skeletal Radiol 2007; 36: 1017-1027.

7. Herneth AM, Dominkus M, Kurtaran A, et al. Bone metastases: new trends in diagnostic imaging. Wien Med Wochenschr Suppl 2002; 113: 92-94.

8. El-Samie HAE-KA, El-Ghany HSA. The value of added opposed/ in phase MRI sequences in characterization of the focal vertebral bone marrow lesions in oncology patients. Egypt J Radiol Nucl Med 2015; 46: 727-732.

9. Tadros MY, Louka AL. Discrimination between benign and malignant in vertebral marrow lesions with diffusion weighted MRI and chemical shift. Egypt J Radiol Nucl Med 2016; 47: 557-569.
10. Disler DG, McCauley TR, Ratner LM, et al. In-phase and out-ofphase MR imaging of bone marrow: prediction of neoplasia based on the detection of coexistent fat and water. AJR Am J Roentgenol 1997; 169: 1439-1447.

11. Mubarak F, Akhtar W. Acute vertebral compression fracture: differentiation of malignant and benign causes by diffusion weighted magnetic resonance imaging. JPMA J Pak Med Assoc 2011; 61: 555-558.

12. Gokalp G, Mutlu FS, Yazici Z, Yildirim N. Evaluation of vertebral bone marrow fat content by chemical-shift MRI in osteoporosis. Skeletal Radiol 2011; 40: 577-585.

13. Baker LL, Goodman SB, Perkash I, et al. Benign versus pathologic compression fractures of vertebral bodies: assessment with conventional spin-echo, chemical-shift, and STIR MR imaging. Radiology 1990; 174: 495-502.

14. Ogura A, Hayakawa K, Maeda F, et al. Differential diagnosis of vertebral compression fracture using in-phase/opposed-phase and short TI inversion recovery imaging. Acta Radiol 2012; 53: 450-455.

15. Zampa V, Cosottini M, Michelassi M, et al. Value of opposed-phase gradient-echo technique in distinguishing between benign and malignant vertebral lesions. Eur Radiol 2002; 12: 1811-1818.

16. Zidan DZ, Habib LA, Chalabi NA. Quantitative chemical-shift MR imaging cutoff value: Benign versus malignant vertebral compression - initial experience. Egypt J Radiol Nucl Med 2014; 45: 779-786.

17. Erly WK, Oh ES, Outwater EK. The utility of in-phase/opposedphase imaging in differentiating malignancy from acute benign compression fractures of the spine. Am J Neuroradiol 2006; 27: 1183-1188. 